\title{
Damping of coronal loop kink oscillations due to mode conversion ${ }^{\star}$
}

\author{
M. S. Ruderman ${ }^{1}$ and J. Terradas ${ }^{2}$ \\ ${ }^{1}$ Solar Physics and Space Plasma Research Centre ( $\left.\mathrm{SP}^{2} \mathrm{RC}\right)$, University of Sheffield, Hicks Building, Hounsfield Road, \\ Sheffield S3 7RH, UK \\ e-mail: m.s.ruderman@sheffield.ac.uk \\ 2 Departament de Física, Universitat de les Illes Balears, 07122 Palma de Mallorca, Spain \\ Received 9 August 2012 / Accepted 24 April 2013
}

\section{ABSTRACT}

\begin{abstract}
The damping of kink oscillations of a thin magnetic tube due to mode conversion, also called resonant absorption, is studied. The tube consists of a homogeneous core region and an inhomogeneous annulus, where the density monotonically decreases from its value in the core region to the value in the surrounding plasma. The annulus is assumed to be thin, so the study is carried out in the thin tube thin boundary approximation. The equation governing the amplitude variation of kink oscillations is derived. The initial value problem for this equation is solved to study the resonant damping. This means that, in particular, we study the transient state before the loop oscillates with the stationary or nearly stationary state. The results are compared with those of the direct numerical modelling, and the agreement is found to be fairly good. On the basis of the solution to the initial value problem for the governing equation, the damping time is calculated and compared with that given by the classical theory of resonant absorption. It is found that the classical theory underestimates the damping time, with the error increasing with the increase of the annulus thickness. However, the error is not large, so the damping time given by the classical theory of resonant absorption can be taken as a sufficiently good approximation.
\end{abstract}

Key words. magnetohydrodynamics (MHD) - Sun: corona - Sun: oscillations

\section{Introduction}

After coronal loop kink oscillations were first observed by Transition Region and Coronal Explorer (TRACE) in 1998 and reported by Aschwanden et al. (1999) and Nakariakov et al. (1999), they remain in the limelight of solar physics. One important property of these oscillations is that they are heavily damped with the damping time of the order of a few oscillation periods. A few mechanisms of this damping were suggested. At present the most reliable and comprehensively studied damping mechanism is resonant absorption that is related to the conversion of a global kink oscillation into local small-scale Alfvénic oscillations. Resonant absorption was suggested as a possible mechanism of damping of (at that time hypothetical) coronal loop kink oscillations by Hollweg \& Yang (1988). Later it was more comprehensively studied by Goossens et al. (1992). Ruderman \& Roberts (2002) studied the first observed event and found that resonant absorption is a viable mechanism of damping of coronal loop kink oscillations. Later Goossens et al. (2002) applied it to 12 events of coronal loop oscillations reported by Ofman \& Aschwanden (2002) and confirmed the conclusion made by Ruderman \& Roberts (2002).

Following earlier studies (see, e.g. Sedláček 1971) of resonant absorption, Hollweg \& Yang (1988) used the ideal magnetohydrodynamic (MHD) equations to describe the resonant damping of kink oscillations. In this approach the damping occurs due to the singularity on the Riemann surface of the Laplace transform of the solution when it is extended from the principal sheet to the non-principal sheets of the Riemann surface.

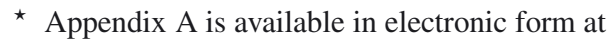
http: //www . aanda.org
Although this approach is mathematically perfectly correct, the occurrence of damping looks like a mathematical trick because all physical processes at the vicinity of the resonant surface are ignored.

Goossens et al. (1992) adopted another approach based on the use of dissipative MHD for the description of wave motion in the vicinity of the resonant surface. To calculate the decrement, they used the connection formulae first introduced by Sakurai et al. (1991). The idea of this approach is the following. Dissipation is important only in a thin dissipative layer embracing the resonant surface because it is characterised by large gradients. The plasma motion can be described by the ideal MHD outside the dissipative layer. The solution of the dissipative MHD equations is used to calculate the jumps of the total pressure and the normal velocity component across the dissipative layer. The magnitudes of these jumps are given by the connection formulae. They are used to connect the ideal MHD solutions at the two sides of the dissipative layer. Sakurai et al. (1991) presented the solution of the dissipative MHD equations in the dissipative layer in terms of Bessel functions. Later Goossens et al. (1995) improved the analysis by Sakurai et al. (1991) and expressed the solution in the dissipative layer in a much simpler form in terms of the so-called $F$ and $G$ functions.

The connection formulae were first used to study the resonant absorption in the driven problem, where the steady state of oscillation is created by an external harmonic driver. Later Goossens et al. (1992) used them to study the damping of viscous eigenmodes in cylindrical geometry. In fact, a similar approach based on matching the ideal MHD solution outside the dissipative layer with the dissipative MHD solution inside it was used by Mok \& Einaudi (1985) to study the damping of surface waves propagating along a transitional layer between two 
homogeneous regions, although these authors did not introduce the connection formulae explicitly.

Mok \& Einaudi (1985) were probably the first who pointed out that the solution in the dissipative layer obtained for the driven problem can be used to describe the eigenmodes of dissipative MHD only when the dissipation is strong enough. To formulate this condition explicitly, we first need to specify the equilibrium. In what follows we consider a straight magnetic tube of radius $R$ and length $L \gg R$. In cylindrical coordinates $r, \varphi, z$ the plasma density is given by

$\rho=\left\{\begin{array}{cc}\rho_{\mathrm{i}}, & r \leq R-\ell / 2, \\ \rho_{\mathrm{t}}(r), & R-\ell / 2 \leq r \leq R+\ell / 2, \\ \rho_{\mathrm{e}}, & r \geq R+\ell / 2,\end{array}\right.$

where $\rho_{\mathrm{i}}$ and $\rho_{\mathrm{e}}$ are constants, $\rho_{\mathrm{i}}>\rho_{\mathrm{e}}$, and $\rho_{\mathrm{t}}(r)$ is a monotonically decreasing function, $\rho_{\mathrm{t}}(R-\ell / 2)=\rho_{\mathrm{i}}, \rho_{\mathrm{t}}(R+\ell / 2)=\rho_{\mathrm{e}}$. We also assume that the transitional layer is thin, $\ell \ll R$. Hence, we use the thin tube thin boundary (TTTB) approximation. We also use the approximation of cold plasma relevant for the coronal conditions.

In the viscous and magnetic Reynolds numbers, $R_{\mathrm{e}}=C_{\mathrm{k}} \ell / v$ and $R_{\mathrm{m}}=C_{\mathrm{k}} \ell / \lambda, v$ is the shear kinematic viscosity, $\lambda$ is the magnetic diffusion coefficient, and $C_{\mathrm{k}}$ is the kink speed determined by

$C_{\mathrm{k}}^{2}=\frac{2 B^{2}}{\mu_{0}\left(\rho_{\mathrm{i}}+\rho_{\mathrm{e}}\right)}$,

where $B$ is the magnitude of the (constant) equilibrium magnetic field and $\mu_{0}$ the magnetic permeability of free space. To characterise the simultaneous effect of viscosity and resistivity, we introduce the total Reynolds number $R_{\mathrm{t}}$ defined by

$\frac{1}{R_{\mathrm{t}}}=\frac{1}{R_{\mathrm{e}}}+\frac{1}{R_{\mathrm{m}}}$

Then the condition that dissipation is strong enough to ensure that the solution in the dissipative layer obtained for the driven problem can be used to describe the eigenmodes of dissipative MHD is written as (e.g. Ruderman et al. 1995)

$R_{\mathrm{t}} \ll(L / \ell)^{4}$.

Ruderman et al. (1995) reconsidered the problem studied by Mok \& Einaudi (1985) when this condition is not satisfied. They showed that, while the solution describing the plasma motion in the dissipative layer is very much different from that obtained for the driven problem, the damping rate of the surface mode remains the same. Later Tirry \& Goossens (1996) extended this analysis to the cylindrical geometry (see also the review paper by Goossens et al. 2011). They expressed the solution in the dissipative layer in terms of $F_{\Lambda}$ and $G_{\Lambda}$ functions, which are the generalisation of $F$ and $G$ functions and coincide with these functions when $\Lambda=0$. Using this solution they showed that the connection formulae remain the same, even when the condition (4) is not satisfied. We note that for typical conditions in the solar corona $R_{\mathrm{t}} \gtrsim(L / \ell)^{4}$.

While the use of connection formulae is perfectly correct when studying the driven problem, their application to the initial value problem is doubtful. The point is that the derivation of connection formulae for the initial value problem is based on the assumption that the plasma motion near the ideal resonant position is described by the $F_{\Lambda}$ and $G_{\Lambda}$ functions. To be specific, we consider the coronal loop kink oscillations. It was shown by Ruderman \& Roberts (2002) that, under not very restrictive assumptions about the initial conditions, the fundamental kink mode of the coronal loop kink oscillation emerges from the initial perturbation after the transitional time of the order of one oscillation period (see also the numerical work by Terradas et al. 2006). However, the plasma motion in this oscillation is described fairly well by the eigenmode of dissipative MHD only far from the resonant surface. Near the resonant surface, the plasma motion is approximately described by the dissipative eigenmode only when the spatial scales of the order of $R_{\mathrm{t}}^{-1 / 3} \ell$ have been formed. This occurs after the transitional time of the order of $R_{\mathrm{t}}^{1 / 3}$ times the oscillation period (e.g. Kappraff \& Tataronis 1977; Poedts et al. 1990; Mann et al. 1995). For typical coronal conditions we have $R_{\mathrm{e}} \sim R_{\mathrm{m}}$, so for the estimates we can take $R_{\mathrm{t}} \sim R_{\mathrm{m}}$. For the coefficient of magnetic diffusion, we have $\lambda=10^{9} T^{-3 / 2} \mathrm{~m}^{2} \mathrm{~s}^{-1}$ (e.g. Priest 1982). Then, taking $C_{\mathrm{k}}=1000 \mathrm{~km} \mathrm{~s}^{-1}$ and $\ell=1000 \mathrm{~km}$ we obtain $R_{\mathrm{m}}=10^{10}$, and we can take $R_{\mathrm{t}}^{1 / 3}=10^{3}$ as a typical value. Hence, the time needed for the formation of spatial scales of the order of $R_{\mathrm{t}}^{-1 / 3} \ell$ is about 1000 oscillation periods. At that time we can expect the spatial scale of the order of $1 \mathrm{~km}$. On the other hand, observations show that the damping of coronal loop kink oscillations occurs after a few oscillation periods. At that time scale, the plasma motion near the resonant surface is quite different from that described by $F_{\Lambda}$ and $G_{\Lambda}$ functions. This means that the main assumption made by Ruderman et al. (1995) and Tirry \& Goossens (1996) when deriving the connection fortmulae is not satisfied.

Recently, the problem of spatial resonant damping of kink waves excited by a driver at the footpoint of a semi-infinite magnetic tube and then propagating along this tube has attracted the attention of solar physicists (Ruderman et al. 2010; Terradas et al. 2010; Soler et al. 2011). In particular, it was shown with the use of connection formulae that in the linear approximation the waves damp exponentially with the distance from the driver, and the damping length has been calculated. The same problem has been studied numerically by Pascoe et al. (2010, 2011, 2012). In particular, Pascoe et al. (2012) have shown that the wave amplitude does not decay exponentially everywhere. At distances of the order of a few wave lengths, the amplitude dependence on the distance is described by the Gaussian profile. At larger distances the amplitude decays exponentially. Motivated by these results, Hood et al. (2013) developed the analytic theory of spatial wave damping that matches very well the results of numerical modelling.

There is a very close similarity between the spatial resonant damping of propagating kink waves and temporal damping of standing kink waves. Hence, we can expect that purely exponential damping does not describe properly the time dependence of the amplitude of kink oscillations. The aim of this paper is to study this problem analytically and then compare the results with the direct numerical modelling. The paper is organised as follows. In the next section we formulate the problem and present the governing equations, as well as the initial and boundary conditions. In Sect. 3 we derive the equation that describes the variation of the kink oscillation amplitude. In Sect. 4 we use this equation to study the damping of kink oscillations due to resonant absorption. Section 5 contains the summary of the results and our conclusions.

\section{Problem formulation and governing equations}

In our analysis we use the equation derived by Ruderman (2011, hereafter Paper I). This is Eq. (23) in Paper I, with its right-hand 
side given by Eq. (24). This equation describes kink oscillations of a straight magnetic tube in the thin tube approximation in cold plasma in the presence of background flow. The tube consists of a core region, whose density does not vary in the radial direction and an annulus surrounding this region, where the density monotonically changes from its value in the core region to its value in the surrounding plasma. If we assume that there is no background, flow then Eq. (23) of Paper I reduces to

$$
\frac{\partial^{2} \eta}{\partial t^{2}}-C_{\mathrm{k}}^{2} \frac{\partial^{2} \eta}{\partial z^{2}}=\frac{\mathcal{D}}{\rho_{\mathrm{i}}+\rho_{\mathrm{e}}}
$$

Here $\eta$ is the radial displacement in the core region, where it is independent of $r$ in cylindrical coordinates $r, \varphi, z$, with the $z$-axis coinciding with the loop axis; $\rho$ is the equilibrium plasma density given by Eq. (1), and $C_{\mathrm{k}}$ is the kink speed given by Eq. (2). The right-hand side of Eq. (5) describes the wave damping due to resonant absorption. The quantity $\mathcal{D}$ is given by

$$
\begin{aligned}
\mathcal{D}= & \frac{\ell \rho_{\mathrm{i}}}{R}\left(\frac{\partial^{2} \eta}{\partial t^{2}}-V_{\mathrm{A} i}^{2} \frac{\partial^{2} \eta}{\partial z^{2}}\right)+\frac{\delta P}{R} \\
& -\rho_{\mathrm{e}}\left(\frac{\partial^{2} \delta \xi_{r}}{\partial t^{2}}-V_{\mathrm{Ae}}^{2} \frac{\partial^{2} \delta \xi_{r}}{\partial z^{2}}\right),
\end{aligned}
$$

where $P=B b_{z} / \mu_{0}$ is the magnetic pressure perturbation, $b_{z}$ is the $z$-component of the magnetic field perturbation, $\xi_{r}$ and $\xi_{\varphi}$ are the plasma displacements in the radial and azimuthal direction, $\delta P$ and $\delta \xi_{r}$ are the jumps of $P$ and $\xi_{r}$ across the annulus, and $V_{\mathrm{A}}$ is the Alfvén speed determined by

$V_{\mathrm{A}}^{2}=\frac{B^{2}}{\mu_{0} \rho}$.

We note that $\xi_{r}=\eta$ for $r \leq R-\ell / 2$. As we have already stated, in the following we use the TTTB approximation and assume that the annulus is thin, $\epsilon=\ell / R \ll 1$. In accordance with this, we will calculate $\mathcal{D}$ only in the leading order approximation with respect to $\epsilon$.

In Paper I the equations describing the plasma motion in terms of the magnetic pressure perturbation $P$ and the plasma displacement $\boldsymbol{\xi}$ are derived (see Eqs. (11) and (12)). It is also pointed out in the paragraph before Eq. (48) in Paper I that the ratio of the left-hand side of Eq. (11) to its right-hand side is $O\left(R^{2} / L^{2}\right)$, so that the right-hand side can be neglected in the thin tube approximation. We would like to study linearly polarised kink oscillations. In accordance with this we take $\xi_{r} \propto$ $\cos \varphi, P \propto \cos \varphi$, and $\xi_{\varphi} \propto-\sin \varphi$. Then Eq. (11) of Paper I with the left-hand side equal to zero and Eq. (12) with $U=0$ (no equilibrium flow) reduce to

$$
\begin{aligned}
& \frac{\partial\left(r \xi_{r}\right)}{\partial r}-\xi_{\varphi}=0, \\
& \frac{\partial^{2} \xi_{r}}{\partial t^{2}}-V_{\mathrm{A}}^{2} \frac{\partial^{2} \xi_{r}}{\partial z^{2}}=-\frac{1}{\rho} \frac{\partial P}{\partial r}, \\
& \frac{\partial^{2} \xi_{\varphi}}{\partial t^{2}}-V_{\mathrm{A}}^{2} \frac{\partial^{2} \xi_{\varphi}}{\partial z^{2}}=-\frac{P}{\rho r} .
\end{aligned}
$$

We assume that the magnetic field lines are frozen in the dense photospheric plasma at the loop footpoints. Hence, we impose the boundary conditions

$\eta=\xi_{r}=\xi_{\varphi}=0 \quad$ at $\quad z=0, L$.

In what follows we restrict our attention to the fundamental mode of kink oscillations and take $\eta, \xi_{r}, \xi_{\varphi}$, and $P$ proportional to $\sin (k z)$, where $k=\pi / L$.
As we have already mentioned, the motion far from the Alfvén resonant surface, in particular, the motion outside the inhomogeneous annulus, is described fairly well by the eigenmode after the transitional time of the order of the oscillation period. Hence, after this transitional time, the plasma motion is the harmonic oscillation with the frequency $\omega=k C_{\mathrm{k}}$ and the amplitude slowly decaying due to resonant absorption. This implies that we can use the approximate relation

$\frac{\mathrm{d}^{2} f}{\mathrm{~d} t^{2}} \approx-\omega^{2} f$

which is valid for $\eta, \xi_{r}$, and $\delta \xi_{r}$. However, this is generally not true for the variation of $\xi_{\varphi}$ inside the inhomogeneous layer. Using the prescribed dependence on $z$ and Eq. (12), we rewrite Eqs. (5), (6), (9), and (10) as

$$
\begin{aligned}
& \frac{\mathrm{d}^{2} \eta}{\mathrm{d} t^{2}}+\omega^{2} \eta=\frac{\mathcal{D}}{\rho_{\mathrm{i}}+\rho_{\mathrm{e}}}, \\
& \mathcal{D}=-\frac{\epsilon}{2} \omega^{2}\left(\rho_{\mathrm{i}}-\rho_{\mathrm{e}}\right) \eta+\frac{\delta P}{R}-\frac{1}{2} \omega^{2}\left(\rho_{\mathrm{i}}-\rho_{\mathrm{e}}\right) \delta \xi_{r}, \\
& \frac{\partial P}{\partial r}=\rho k^{2}\left(C_{\mathrm{k}}^{2}-V_{\mathrm{A}}^{2}\right) \xi_{r}, \\
& \frac{\partial^{2} \xi_{\varphi}}{\partial t^{2}}+k^{2} V_{\mathrm{A}}^{2} \xi_{\varphi}=-\frac{P}{\rho r} .
\end{aligned}
$$

Equation (8) does not change.

We also need to impose the initial conditions. We assume that at the initial moment of time the loop is pushed by an external pulse to oscillate with the fundamental frequency. This implies, in particular, that the initial conditions for $\eta$ are

$\eta=0, \quad \frac{\mathrm{d} \eta}{\mathrm{d} t}=a \omega \quad$ at $\quad t=0$,

where $a$ is a positive constant. In the homogeneous core $\xi_{\varphi}=$ $\eta$, so $\partial \xi_{\varphi} / \partial t=a \omega$ at $t=0$. Outside the inhomogeneous layer $(r>R+\ell / 2) \xi_{\varphi}=-\eta / r^{2}$. We assume that in the inhomogeneous transitional layer

$\xi_{\varphi}=0, \quad \frac{\partial \xi_{\varphi}}{\partial t}=a \omega \operatorname{sgn}(R-r) \quad$ at $\quad t=0$.

Equations (13)-(16), together with the initial conditions (17) and (18), will be used in the next section to derive the equation for $\eta$.

\section{Derivation of the governing equation for $\eta$}

In order to derive the governing equation for $\eta$, we need to express $\mathcal{D}$ in terms of $\eta$. As we have already mentioned, in the homogeneous core $\xi_{r}$ is independent of $r$, so $\xi_{r}=\eta$ for $r \leq R-\ell / 2$. It is shown in Paper I (see Eq. (17)) that $P$ is a linear homogeneous function of $r$ and $\delta \xi_{r}=O(\epsilon)$ (see the paragraph before Eq. (20) in Paper I). Then it follows from Eq. (15) that

$P_{\mathrm{i}}=\frac{1}{2} \omega^{2} R\left(\rho_{\mathrm{i}}-\rho_{\mathrm{e}}\right) \eta+O(\epsilon)$,

where $P_{\mathrm{i}}=P$ at $r=R-\ell / 2$. It also follows from Eq. (17) of Paper I that

$\delta P \approx \epsilon P_{\mathrm{i}}$.

Using Eqs. (19) and (20), we can see that the first and second term in Eq. (14) cancel each other. 
The next step is to express $\delta \xi_{r}$ in terms of $\eta$. For this we first need to solve Eq. (16) with respect to $\xi_{\varphi}$. Using Eqs. (19) and (20), we rewrite Eq. (16) in the approximate form valid in the inhomogeneous annulus,

$\frac{\partial^{2} \xi_{\varphi}}{\partial t^{2}}+k^{2} V_{\mathrm{A}}^{2} \xi_{\varphi}=-\frac{\omega^{2}}{2 \rho_{\mathrm{t}}}\left(\rho_{\mathrm{i}}-\rho_{\mathrm{e}}\right) \eta$

The solution to this equation satisfying the initial conditions (18) is

$$
\begin{aligned}
\xi_{\varphi}= & \frac{a \omega}{k V_{\mathrm{A}}} \operatorname{sgn}(R-r) \sin \left(k V_{\mathrm{A}} t\right) \\
& -\kappa k V_{\mathrm{A}} \int_{0}^{t} \eta\left(t-t^{\prime}\right) \sin \left(k V_{\mathrm{A}} t^{\prime}\right) \mathrm{d} t^{\prime},
\end{aligned}
$$

where

$\kappa=\frac{\rho_{\mathrm{i}}-\rho_{\mathrm{e}}}{\rho_{\mathrm{i}}+\rho_{\mathrm{e}}}$.

In what follows we also assume that the density profile in the inhomogeneous layer is linear, so

$\rho_{\mathrm{t}}(r)=\frac{1}{\ell}\left[\rho_{\mathrm{i}}(R+\ell / 2-r)-\rho_{\mathrm{e}}(R-\ell / 2-r)\right]$.

Using Eqs. (8) and (22), we then obtain

$$
\begin{aligned}
\delta \xi_{r} & =\frac{1}{R} \int_{R-\ell / 2}^{R+\ell / 2} \xi_{\varphi} \mathrm{d} r \\
& =\frac{\epsilon a}{\kappa} F(\omega t)-\epsilon \omega \int_{0}^{t} G\left(\omega t^{\prime}\right) \eta\left(t-t^{\prime}\right) \mathrm{d} t^{\prime},
\end{aligned}
$$

where

$$
\begin{aligned}
F(x)= & -\frac{x}{6}\left(2 \cos x-(1+\kappa) \cos \frac{x}{\sqrt{1+\kappa}}\right. \\
& \left.-(1-\kappa) \cos \frac{x}{\sqrt{1-\kappa}}\right)+\frac{x^{2}-2}{6}(2 \sin x \\
& \left.-\sqrt{1+\kappa} \sin \frac{x}{\sqrt{1+\kappa}}-\sqrt{1-\kappa} \sin \frac{x}{\sqrt{1-\kappa}}\right) \\
& +\frac{\kappa}{3}\left(\sqrt{1+\kappa} \sin \frac{x}{\sqrt{1+\kappa}}-\sqrt{1-\kappa} \sin \frac{x}{\sqrt{1-\kappa}}\right) \\
& -\frac{x^{3}}{6}\left(2 \mathrm{Ci}(x)-\mathrm{Ci} \frac{x}{\sqrt{1+\kappa}}-\mathrm{Ci} \frac{x}{\sqrt{1-\kappa}}\right), \\
G(x)= & \sqrt{1+\kappa} \sin \frac{x}{\sqrt{1+\kappa}}-\sqrt{1-\kappa} \sin \frac{x}{\sqrt{1-\kappa}} \\
& -x \mathrm{Ci} \frac{x}{\sqrt{1+\kappa}}+x \operatorname{Ci} \frac{x}{\sqrt{1-\kappa}},
\end{aligned}
$$

and $\operatorname{Ci}(x)$ is the integral cosine defined by

$\operatorname{Ci}(x)=-\int_{x}^{\infty} \frac{\cos u}{u} \mathrm{~d} u=\ln x+C-\int_{0}^{x} \frac{1-\cos u}{u} \mathrm{~d} u$,

with $C \approx 0.5772$ being the Euler constant. Using Eq. (25), we can express $\mathcal{D}$ in terms of $\eta$. Substituting the obtained result in Eq. (13), we eventually arrive at the governing equation for $\eta$,

$\frac{\mathrm{d}^{2} \eta}{\mathrm{d} t^{2}}+\omega^{2} \eta=\frac{\epsilon \kappa \omega^{3}}{2} \int_{0}^{t} G\left(\omega t^{\prime}\right) \eta\left(t-t^{\prime}\right) \mathrm{d} t^{\prime}-\frac{\epsilon R \omega^{2}}{2} F(\omega t)$.

This integro-differential equation describes the evolution of the tube displacement with time. In the next section it will be used to study resonant damping of kink oscillations.

\section{Resonant damping of kink oscillations}

First we discuss the asymptotic behaviour of the solution to Eq. (29) for large $t$. It follows from Eq. (A.23) in Appendix A that for $\pi \epsilon \omega t / 8 \sim 1$ the oscillation amplitude is proportional to $\mathrm{e}^{-\gamma_{\mathrm{d}} t}$, where

$\gamma_{\mathrm{d}}=\frac{\pi \epsilon \kappa \omega}{8}=\frac{\pi^{2} \ell\left(\rho_{\mathrm{i}}-\rho_{\mathrm{e}}\right)}{4 R \Pi\left(\rho_{\mathrm{i}}+\rho_{\mathrm{e}}\right)}$,

and $\Pi=2 \pi / \omega$ is the wave period. This is a familiar result (see, e.g. Goossens et al. 1992, 2002; Ruderman \& Roberts 2002). We have imposed the restriction $\pi \epsilon \omega t / 8 \sim 1$ and do not consider a larger time, $\omega t \gg \epsilon^{-1}$, because in accordance with Eq. (A.23) Eq. (29) describes the exponential decay of oscillation amplitude only for $t \sim 8(\pi \epsilon \omega)^{-1}$. For $\omega t \gg \epsilon^{-1}$, the term $O(1 / \omega t)$ on the right-hand side of Eq. (A.23) is either comparable with the exponential term or even dominant. Hence, it seems that Eq. (29) is valid only for $\pi \epsilon \omega t / 8 \lesssim 1$.

Equation (A.11) expresses the solution of Eq. (29) in terms of integral along the real axis. It can be used to study the dependence of $\eta$ on time. However, we preferred to solve Eq. (29) numerically. To do this we approximated the derivative on the left-hand side of this equation by the central difference and used the trapezoid rule to calculate the integral on the right-hand side. As a complementary analysis we have also solved the problem numerically by using the original linearised MHD equations and applying the initial conditions given by Eqs. (17), (18) (see Terradas et al. 2006, for further details). This allows us to compare the performance of the two methods. The results of the numerical solution of Eq. (29) and the direct numerical modelling for various values of $\epsilon$ are shown in Fig. 1-6. In all calculations we took $\zeta=\rho_{\mathrm{i}} / \rho_{\mathrm{e}}=3$, so that $\kappa=1 / 2$. Figures $1-3$ show the solution of Eq. (29), together with solutions obtained by the direct numerical modelling for $\epsilon=0.1,0.2,0.3$. In what follows we define the damping time $t_{\mathrm{d}}$ as the time when the oscillation amplitude decreases $e$ times in comparison with its initial value. In Figs. 1-3 the solutions are shown on the time interval approximately equal to $2 t_{\mathrm{d}}$. We note that, while the solid and dashed curve in each figure practically coincide for first few periods, the amplitude of the solid curve becomes slightly smaller than that of the dashed curve for large time. We attribute this difference to the approximations made in the derivation of Eq. (29). This comparison gives further support to the conclusion that Eq. (29) is valid only for $\omega t \lesssim \epsilon^{-1}$. We also note the small difference in period as $\epsilon$ increases; this is because the direct numerical results are not based on the thin tube assumption (nor the thin boundary approximation) and the inhomogeneous distribution in the layer produces an increase in the period of oscillation.

Examination of Figs. 1-3 reveals that the solution can be written in the form $\eta(t)=A(t) \sin (\omega t+\Phi(t))$. The phase shift $\Phi(t)$ is very small and we did not study it. Our attention was concentrated on the behaviour of the wave amplitude $A(t)$, which is displayed in Figs. 4-6. In these figures the solid lines show $\ln (A / R)$ calculated using Eq. (29). The circles show the same quantity calculated using the direct numerical modelling. We see that for small $t / \Pi$ the curves showing the dependence of $\ln (A / R)$ on time have a parabolic shape, while they are approximately linear for larger values of $t / \Pi$. The distance where the transition from the parabolic to linear shape occurs increases with the increase of $\epsilon$. Hence the oscillation amplitude is described by a kind of Gaussian profile for small time and by the exponential function for larger time. This result is similar to one found by Pascoe et al. $(2010,2011,2012)$ for propagating waves. The dashed line in 
M. S. Ruderman and J. Terradas: Damping of coronal loop kink oscillations

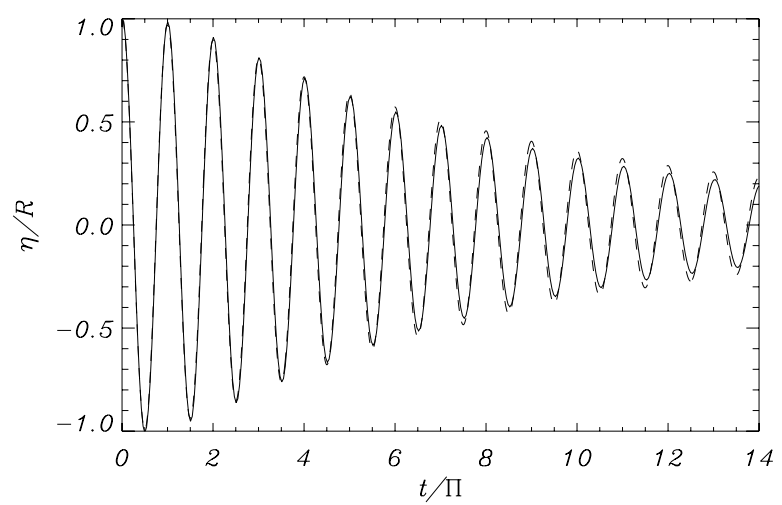

Fig. 1. Dependence of $\eta$ on time found by the direct numerical modelling (solid line) and by solving Eq. (29) (dashed line) for $\epsilon=0.1$.

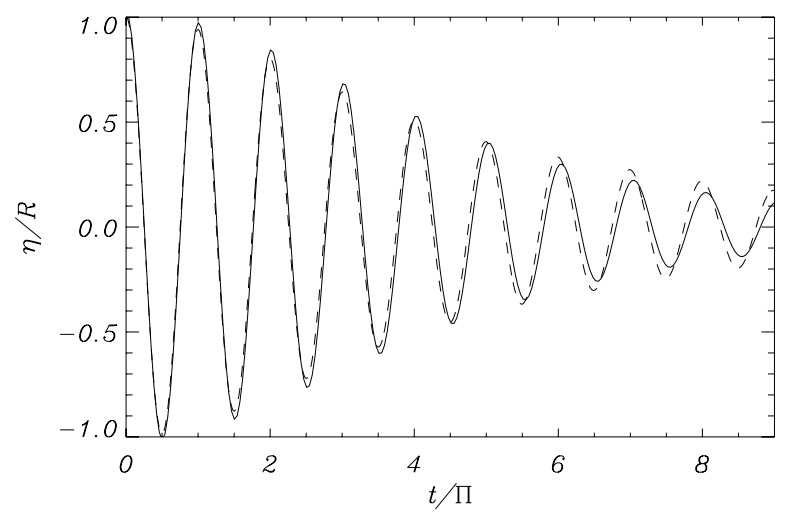

Fig. 2. Same as Fig. 1, but for $\epsilon=0.2$.

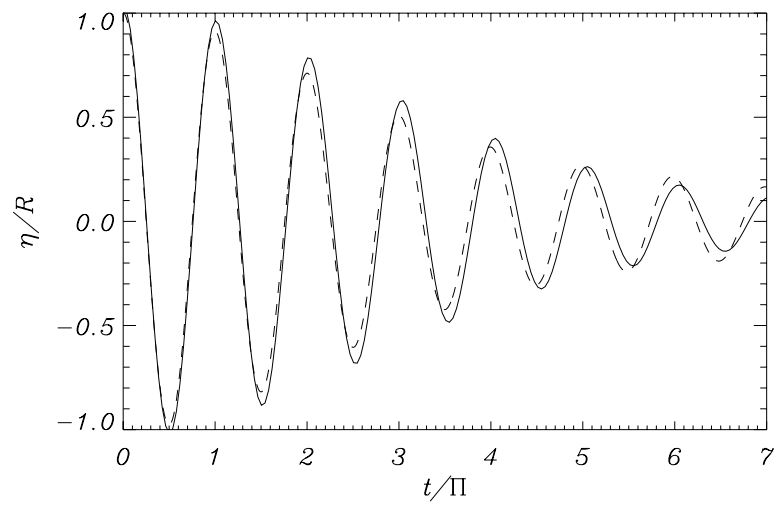

Fig. 3. Same as Fig. 1, but for $\epsilon=0.3$.

Figs. 4-6 show $\ln (A / R)$ given by the classical theory of resonant absorption.

We calculate the damping time $t_{\mathrm{d}}$ defined by $A\left(t_{\mathrm{d}}\right) / R=\mathrm{e}^{-1}$ and compare it with the damping time predicted by the classical theory of resonant absorption, $t_{\mathrm{dc}}=1 / \gamma_{\mathrm{d}}$, where $\gamma_{\mathrm{d}}$ is given by Eq. (30). It follows both from the direct numerical modelling and from the solution of Eq. (29) that $t_{\mathrm{d}} / \Pi \approx 8.8$ for $\epsilon=0.1, t_{\mathrm{d}} / \Pi \approx 4.6$ for $\epsilon=0.2$, and $t_{\mathrm{d}} / \Pi \approx 3.2$ for $\epsilon=0.3$. Using Eq. (30) we obtain $t_{\mathrm{dc}} / \Pi=4\left(\pi^{2} \epsilon \kappa\right)^{-1}$. As a result we find that $t_{\mathrm{d}}$ exceeds $t_{\mathrm{dc}}$ by about $9 \%$ for $\epsilon=0.1$, about $14 \%$ for $\epsilon=0.2$, and about $18 \%$ for $\epsilon=0.3$. Hence, we conclude that the classical theory of resonant absorption underestimates the damping time and that the error grows when $\epsilon$ increases. However, the error is not very large, so the damping time given

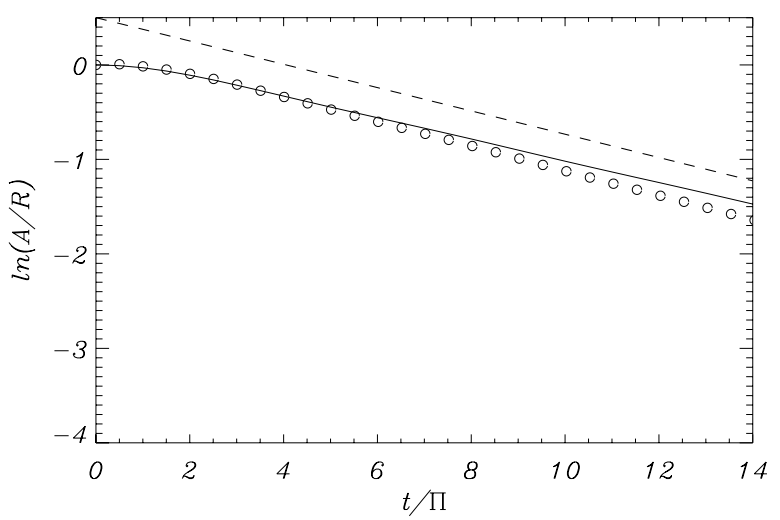

Fig. 4. Dependence of $\ln (A / R)$ on time for $\epsilon=0.1$. The solid line corresponds to the solution of Eq. (29). The circles show the result of the direct numerical modelling. The dashed line shows the linear dependence for large $t$.

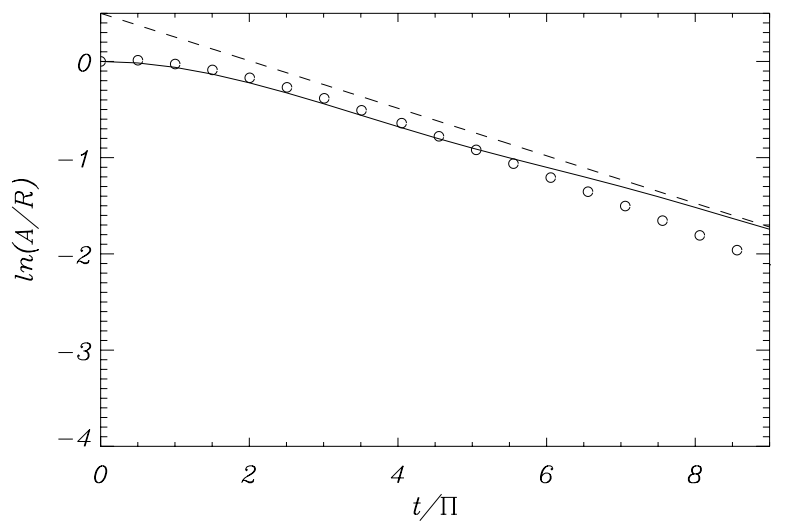

Fig. 5. Same as Fig. 4, but for $\epsilon=0.2$.

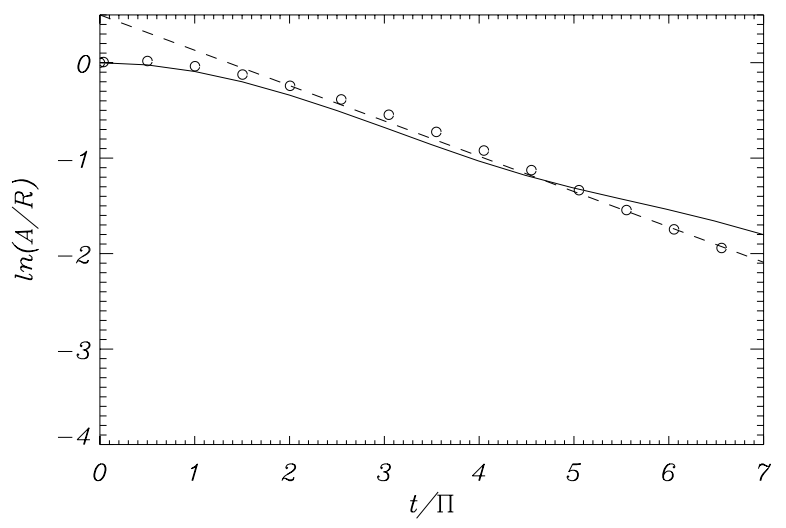

Fig. 6. Same as Fig. 4, but for $\epsilon=0.3$.

by the classical theory of resonant absorption can be taken as a reasonable approximation.

It is instructive to compare the results obtained in this paper with those obtained by Hood et al. (2013). These authors studied the resonant damping of propagating kink waves in a semiinfinite magnetic tube, where the waves are excited by a driver at the tube end. There is an obvious similarity between our results and those obtained by Hood et al. (2013). They found that the wave amplitude is approximately described by the Gaussian function at sufficiently small distance from the tube end, and we found that the oscillation amplitude is approximately described 
by the Gaussian function at sufficiently small time. Next, Hood et al. (2013) found that at larger distances the wave amplitude decays exponentially and that the distance where the transition from the Gaussian to the exponential amplitude profile occurs increases with the $\epsilon$ increase. We found that at larger time the oscillation amplitude decays exponentially and that the time when the transition from the Gaussian to the exponential amplitude profile occurs increases with the $\epsilon$ increase. Finally, Hood et al. (2013) found that the classical theory of resonant absorption underestimates the damping distance, while we found that it underestimates the damping time.

\section{Summary and conclusions}

In this paper we have studied the resonant damping of kink oscillations of a magnetic tube with the plasma density varying in the radial direction. The TTTB approximation and the approximation of cold plasmas were used. We derived the governing equation for the tube displacement (Eq. (29)).

To study the resonant damping of kink oscillations, we solved Eq. (29) numerically for various values of the ratio $\epsilon$. This ratio compares the thickness of the inhomogeneous layer, where the plasma density varies, to the tube radius. We found that for sufficiently small time the amplitude decrease is described by a kind of Gaussian function, while it becomes exponential for larger time. The larger the $\epsilon$, the later the transition from the Gaussian to the exponential regime occurs. The solutions to Eq. (29) were compared with the results of the direct numerical modelling, and the agreement was found to be fairly good.

We defined the damping time as the time when the oscillation amplitude decreases $e$ times in comparison with its initial value. Then we calculated the damping time using Eq. (29) and compared it with that given by the classical theory of resonant absorption. We found that the classical theory underestimates the damping time, with the error getting larger when $\epsilon$ increases. However, this error is not very large. Even for $\epsilon=0.3$, which is the largest value used in our study, the error is only about $18 \%$. Hence, we conclude that the damping time given by the classical theory can be used as a reasonable approximation.

Acknowledgements. M.S.R. acknowledges the support by a Royal Society Leverhulme Trust Senior Research Fellowship and by an STFC grant. J.T. acknowledges support from the Spanish Ministerio de Educación y Ciencia through a Ramón y Cajal grant and financial support from MICINN/MINECO and FEDER Funds through grant AYA2011-22846 Funding from CAIB through the "Grups Competitius" scheme and FEDER Funds is also acknowledged.

\section{References}

Aschwanden, M. J., Fletcher, L., Schrijver, C. J., \& Alexander, D. 1999, ApJ, 520,880

Goossens, M., Hollweg, J. V., \& Sakurai, T. 1992, Sol. Phys., 138, 233

Goossens, M., Ruderman, M. S., \& Hollweg, J. V. 1995, Sol. Phys., 157, 75

Goossens, M., Andries, J., \& Aschwanden, M. J. 2002, A\&A, 394, L39

Goossens, M., Erdélyi, R., \& Ruderman, M. S. 2011, Space Sci. Rev., 158, 289

Hollweg, J. V., \& Yang, G. 1988, J. Geophys. Res., 93, 5423

Hood, A. W., Ruderman, M. S., De Moortel, I., et al. 2013, A\&A, 551, A39

Kappraff, J. M., \& Tataronis, J. A. 1977, J. Plasma Phys., 18, 209

Mann, I. R., Wright, A. N., \& Cally, P. S. 1995, J. Geophys. Res., 100, 19441

Mok, Y., \& Einaudi, G. 1985, J. Plasma Phys., 33, 199

Nakariakov, V. M., Ofman, L., Deluca, E. E., Roberts, B., \& Davila, J. M. 1999, Science, 285, 862

Pascoe, D. J., Wright, A. N., \& De Moortel, I. 2010, ApJ, 711, 990

Pascoe, D. J., Wright, A. N., \& De Moortel, I. 2011, ApJ, 731, 73

Pascoe, D. J., Hood, A. W., De Mortel, I., \& Wright, A. N. 2012, ApJ, 539, A37 Ofman, L., \& Aschwanden, M. J. 2002, ApJ, 576, L153

Poedts, S., Goossens, M., \& Kerner, W. 1990, Comput. Phys. Commun., 59, 95

Priest, E. 1982, Solar Magneto-Hydrodynamics, Geophysics and Astrophysics

Monographs (Kluwer Academic Publishers)

Ruderman, M. S. 2011, A\&A, 534, A78

Ruderman, M. S., \& Roberts, B. 2002, ApJ, 577, 475

Ruderman, M. S., Tirry, W., \& Goossens, M. 1995, J. Plasma Phys., 54, 129

Ruderman, M. S., Goossens, M., \& Andries, J. 2010, Phys. Plasmas, 17, 082108

Sakurai, T., Goossens, M., \& Hollweg, J. V. 1991, Sol. Phys., 133, 227

Sedláček, Z. 1971, J. Plasma Phys., 5, 239

Soler, R., Terradas, J., \& Goossens, M. 2011, ApJ, 734, 80

Terradas, J., Oliver, R., \& Ballester, J. L. 2006, ApJ, 642, 533

Terradas, J., Goossens, M., \& Verth, G. 2010, A\&A, 524, A23

Tirry, W. J., \& Goossens, M. 1996, ApJ, 471, 501 


\section{Appendix A: Asymptotic solution for large time}

In this appendix we study the asymptotic behaviour of the solution to Eq. (29) for $\omega t \sim \epsilon^{-1}$. We introduce the Laplace transform

$\mathcal{L}[f(t)](s)=\int_{0}^{t} f(t) \mathrm{e}^{\mathrm{i} s t} \mathrm{~d} t$.

Applying the Laplace transform to Eq. (29) and using the theorem about the Laplace transform of convolution, we obtain

$\mathcal{L}[\eta(t)](s)=R \omega \frac{2-\epsilon \mathcal{L}[F(t)](s / \omega)}{2\left(\omega^{2}-s^{2}\right)-\epsilon \kappa \omega^{2} \mathcal{L}[G(t)](s / \omega)}$.

Using the standard table of Laplace transforms, we find

$\mathcal{L}[G(t)](s)=\frac{1}{2 s^{2}} \ln \frac{1-s^{2}(1-\kappa)}{1-s^{2}(1+\kappa)}$.

We do not give the expression for $\mathcal{L}[F(t)](s)$ because it is not used in what follows. The solution to Eq. (29) with the initial condition (17) is given by

$\eta(t)=\frac{R}{2 \pi} \int_{i \varsigma^{-\infty}}^{i \zeta+\infty} \frac{\{2+\epsilon \mathcal{L}[F(t)](s)\} \mathrm{e}^{-\mathrm{i} s \omega t} \mathrm{~d} s}{2-2 s^{2}-\epsilon \kappa \mathcal{L}[G(t)](s)}$,

where $\varsigma$ is any positive constant. When calculating the inverse Laplace transform, we have made the substitution $s=\omega s^{\prime}$ and then dropped the prime.

Now we use Eq. (A.4) to obtain the asymptotic expression for $\eta(t)$ valid for $\omega t \sim \epsilon^{-1}$. The ratio of the second term in the numerator of the integrand in Eq. (A.4) to the first one is of the order of $\epsilon$. Hence, we can neglect the second term in the numerator and use the approximate expression

$\eta(t)=\frac{R}{\pi} \int_{i \varsigma^{-\infty}}^{i \zeta^{+\infty}} \frac{\mathrm{e}^{-\mathrm{i} s \omega t} \mathrm{~d} s}{2-2 s^{2}-\epsilon \kappa \mathcal{L}[G(t)](s)}$

to calculate the asymptotic behaviour of $\eta(t)$ at large time. The integrand in this expression has four logarithmic branch points at $s= \pm s_{+}$and $s= \pm s_{-}$, where

$s_{+}=\frac{1}{\sqrt{1+\kappa}}, \quad s_{-}=\frac{1}{\sqrt{1-\kappa}}$.

To obtain a single-valued branch of the integrand, we make the cuts of the complex $s$-plane along the intervals $\left[-s_{-},-s_{+}\right]$ and $\left[s_{+}, s_{-}\right]$. The multivaluedness of the integrand is related to the presence of logarithm in Eq. (A.3). We define the principal sheet of the Riemann surface of the integrand by the condition that the imaginary part of this logarithm is between $-\pi \mathrm{i}$ and $\pi \mathrm{i}$. In what follows we use the notation "In" for this value of logarithm. All other values differ from this value by $2 \pi n i$, where $n$ is an integer number.

The function

$w(s)=\ln \frac{1-s^{2}(1-\kappa)}{1-s^{2}(1+\kappa)}$

maps the complex $s$-plane with cuts $\left[-s_{-},-s_{+}\right]$and $\left[s_{+}, s_{-}\right]$on the strip $-\pi<\mathfrak{J}(w)<\pi$ in the complex $w$-plane, where $\mathfrak{J}$ indicates the imaginary part of a quantity. We find the zeros of the denominator of the integrand in Eq. (A.5), which are determined by the equation

$\epsilon \kappa w(s)=4 s^{2}\left(1-s^{2}\right)$.

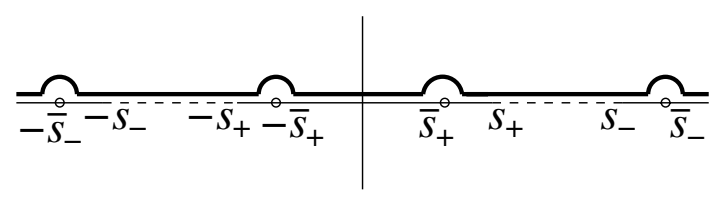

Fig. A.1. Integration contour used to obtain Eq. (A.11) is shown by the thick solid line. The dashed lines show the cuts.

Let $s_{*}$ be a root of this equation. If it is not close to $\pm s_{ \pm}$ then it follows that $s_{*}^{2}\left(1-s_{*}^{2}\right)=O(\epsilon)$. This implies that either $s_{*}=O\left(\epsilon^{1 / 2}\right)$ or $s_{*}= \pm 1+O(\epsilon)$. In the first case we immediately see that the left-hand side of Eq. (A.8) is of the order of $\epsilon^{2}$, so that the only possible solution of this kind is $s_{*}=0$. Since $\mathcal{L}[G(t)](0)=\kappa$, it is straightforward to see that the denominator of the integrand in Eq. (A.5) does not equal zero when $s=0$. Hence, $s_{*}=0$ is a spurious root.

Considering the second possibility, namely, $s_{*}= \pm 1+O(\epsilon)$, we look for the root to Eq. (A.8) in the form $s_{*}= \pm 1+\epsilon \delta$. By substituting this expression in Eq. (A.8) we obtain

$\kappa \ln \left(-1 \pm \frac{4 \epsilon \delta}{\kappa}\right)=\mp 8 \delta+O(\epsilon)$.

The imaginary part of the left-hand side of this equation is close to $-\pi \mathrm{i}$ when $\mathfrak{J}( \pm \delta)<0$ and to $\pi \mathrm{i}$ when $\mathfrak{J}( \pm \delta)>0$. This implies that the imaginary parts of the left- and right-hand side of Eq. (A.9) have different signs, so that this equation cannot have a solution with $\mathfrak{J}(\delta) \neq 0$. It also cannot have a real solution because in that case the imaginary part of the right-hand side is zero, while the imaginary part of the left-hand side is non-zero.

Hence, Eq. (A.8) can only have solutions close to $\pm s_{ \pm}$. We look for the solution in the form $s_{*}= \pm s_{ \pm}+\delta$, where $\delta \ll 1$. Substituting this expression in Eq. (A.8) we obtain four roots, $s= \pm \bar{s}_{+}$and $s= \pm \bar{s}_{-}$, where

$\bar{s}_{ \pm}=\frac{1}{\sqrt{1 \pm \kappa}}\left[1 \mp \frac{\kappa}{1 \pm \kappa} \exp \left(-\frac{4}{\epsilon(1 \pm \kappa)^{2}}\right)\right]$.

Hence, there are four poles on the real axis, at $s= \pm \bar{S}_{+}$and $s=$ $\pm \bar{S}_{-}$.

The integration contour in Eq. (A.5) is a straight line parallel to the real axis. Its equation in $\mathfrak{J}(s)=\varsigma>0$. Since the integrand in Eq. (A.5) is an analytical function in the upper part of the complex $s$-plane, we can deform the integration contour arbitrarily without changing the value of the integral. The only conditions that have to be satisfied are that the new contour is completely in the union of the upper part of the complex $s$-plane and the real axis and that the part of the new contour that is on the real axis does not contain singularities of the integrand. It is convenient to use the new integration contour shown in Fig. A.1. This contour consists of five intervals of the real axis, $\left(-\infty,-\bar{s}_{-}-\varepsilon\right],\left[-\bar{s}_{-}+\varepsilon,-\bar{s}_{+}-\varepsilon\right],\left[-\bar{s}_{+}+\varepsilon, \bar{s}_{+}-\varepsilon\right]$, $\left[\bar{s}_{+}+\varepsilon, \bar{s}_{-}-\varepsilon\right]$, and $\left[\bar{s}_{-}+\varepsilon, \infty\right)$, and four half-circles of radius $\varepsilon$ centred at $\pm \bar{s}_{+}$and $\pm \bar{s}_{-}$. Taking $\varepsilon \rightarrow+0$ we obtain

$$
\begin{aligned}
\eta(t)= & \frac{R}{\pi}\left(\mathcal{P} \int_{-\infty}^{-s_{-}}+\int_{-s_{-}}^{-s_{+}}+\mathcal{P} \int_{-_{+}}^{s_{+}}+\int_{s_{+}}^{s_{-}}+\mathcal{P} \int_{s_{-}}^{\infty}\right) \\
& \times \frac{\mathrm{e}^{-\mathrm{i} s \omega t} \mathrm{~d} s}{2-2 s^{2}-\epsilon \kappa \mathcal{L}[G(t)](s)} \\
& -\frac{\mathrm{i} R}{2} \sum_{j=1}^{4} \operatorname{res}_{s=s_{j}} \frac{\mathrm{e}^{-\mathrm{i} s \omega t}}{2-2 s^{2}-\epsilon \kappa \mathcal{L}[G(t)](s)},
\end{aligned}
$$

where $s_{1,2}= \pm \bar{s}_{+}, s_{3,4}= \pm \bar{s}_{-}, \mathcal{P}$ indicates the principal Cauchy part of an integral, and in the integrals along the 
intervals $\left[-s_{-},-s_{+}\right]$and $\left[s_{+}, s_{-}\right]$the integrand is calculated at the upper edges of the cuts.

First of all, we note that

$\operatorname{res}_{s=s_{j}} \frac{\mathrm{e}^{-\mathrm{i} s \omega t}}{2-2 s^{2}-\epsilon \kappa \mathcal{L}[G(t)](s)}=A_{j} \exp \left(-C_{j} / \epsilon\right)$,

where $j=1,2,3,4, A_{j}$ and $C_{j}$ are constants of the order of unity, and $C_{j}>0$. This implies that the contribution of the last term in Eq. (A.11) is exponentially small and can be neglected. Using the integration by parts we obtain

$\mathcal{P} \int_{-\infty}^{-s_{-}} \frac{\mathrm{e}^{-\mathrm{i} s \omega t} \mathrm{~d} s}{2-2 s^{2}-\epsilon \kappa \mathcal{L}[G(t)](s)}=$

$\frac{\mathrm{i}}{\omega t}\left[\frac{\mathrm{e}^{\mathrm{i} \omega t s_{-}}}{2+2 s_{-}^{2}-\epsilon K \mathcal{L}[G(t)]\left(s_{-}\right)}\right.$

$\left.-\int_{-\infty}^{-s_{-}} \mathrm{e}^{-\mathrm{i} s \omega t} \frac{\mathrm{d}}{\mathrm{d} s}\left(\frac{1}{2-2 s^{2}-\epsilon \kappa \mathcal{L}[G(t)](s)}\right) \mathrm{d} s\right]$.

We see that the contribution of the integral along the interval $\left(-\infty,-s_{-}\right]$is of the order of $1 / \omega t$. Similarly we can show that the contribution of the integrals along the intervals $\left[-s_{+}, s_{+}\right]$ and $\left[s_{-}, \infty\right)$ are also of the order of $1 / \omega t$. Hence, we obtain

$$
\begin{aligned}
\eta(t)= & \frac{R}{\pi}\left(\int_{-s_{-}}^{-s_{+}}+\int_{s_{+}}^{s_{-}}\right) \frac{\mathrm{e}^{-\mathrm{i} s \omega t} \mathrm{~d} s}{2-2 s^{2}-\epsilon \kappa \mathcal{L}[G(t)](s)} \\
& +O(1 / \omega t) .
\end{aligned}
$$

To evaluate the asymptotic behaviour of the integrals in this expression, we need to make the analytic continuation of the function $w(s)$ on its Riemann surface. This Riemann surface consists of an infinite set of complex planes with cuts $\left[-s_{-},-s_{+}\right]$ and $\left[s_{+}, s_{-}\right]$properly attached to each other at the cuts. In what follows we use only two non-principal sheets of the Riemann surface attached to the principal sheet at the upper edges of the cuts. To define $w(s)$ on the non-principal sheet of the Riemann surface attached to the principal sheet at the upper edge of the right cut, we consider a point $s$ near this cut on the principal sheet. We write $s=s_{\mathrm{r}}+\mathrm{i} s_{\mathrm{i}}$, where $s_{+}<s_{\mathrm{r}}<s_{-}$and $\left|s_{\mathrm{i}}\right| \ll s_{\mathrm{r}}$. Then

$$
\begin{aligned}
\frac{1-s^{2}(1-\kappa)}{1-s^{2}(1+\kappa)} \approx & -\frac{(1-\kappa)\left(s_{-}^{2}-s_{\mathrm{r}}^{2}\right)}{(1+\kappa)\left(s_{\mathrm{r}}^{2}-s_{+}^{2}\right)} \\
& +\frac{4 \mathrm{i} \kappa s_{\mathrm{r}} s_{\mathrm{i}}}{(1+\kappa)^{2}\left(s_{\mathrm{r}}^{2}-s_{+}^{2}\right)^{2}}
\end{aligned}
$$

and we obtain

$w\left(s_{\mathrm{r}}+\mathrm{i} s_{\mathrm{i}}\right) \approx \ln \frac{(1-\kappa)\left(s_{-}^{2}-s_{\mathrm{r}}^{2}\right)}{(1+\kappa)\left(s_{\mathrm{r}}^{2}-s_{+}^{2}\right)}+\pi \mathrm{i} \operatorname{sgn}\left(s_{\mathrm{i}}\right)$.

We assume that a point $s$ on the principal Riemann sheet moves in such a way that it crosses the right cut from the upper to the lower part of the complex plane, i.e. $\mathfrak{J}(s)$ changes the sign from positive to negative. Then Eq. (A.16) implies that $\mathfrak{J}(w)$ jumps by $-2 \pi$ i during this crossing. This means that to have the continuous change of $w(s)$ when $s$ moves from the upper part of the principal Riemann sheet to the lower part of the non-principal Riemann sheet we have to define $w(s)$ on this non-principal sheet as

$w(s)=\ln \frac{1-s^{2}(1-\kappa)}{1-s^{2}(1+\kappa)}+2 \pi \mathrm{i}$.

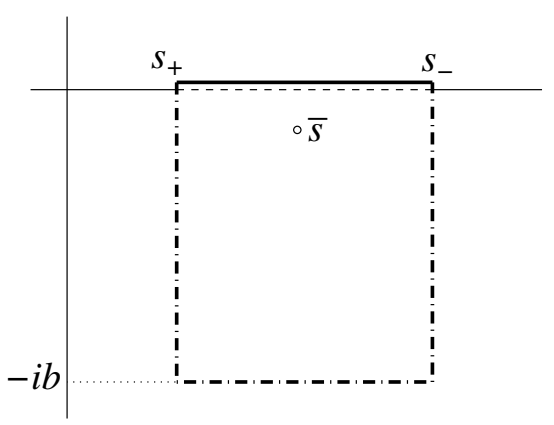

Fig. A.2. Integration contour used to calculate the integral along the interval $\left[s_{+}, s_{-}\right]$in Eq. (A.14). The solid line indicates the part of the contour that coincides with the upper edge of the right cut on the principal Riemann sheet. The dashed-dotted line indicates the parts of the contour on the non-principal Riemann sheet. The dashed line shows the cut.

In a similar way we find that the analytic continuation of $w(s)$ on the non-principal Riemann sheet attached to the principal sheet at the upper edge at the left cut is defined by

$w(s)=\ln \frac{1-s^{2}(1-\kappa)}{1-s^{2}(1+\kappa)}-2 \pi \mathrm{i}$.

To calculate the integral along the interval $\left[s_{+}, s_{-}\right]$in Eq. (A.14), we use the contour shown in Fig. A.2. It consists of the interval $\left[s_{+}, s_{-}\right]$at the upper edge of the right cut on the principal Riemann sheet, two vertical lines from $s_{+}$to $s_{+}-\mathrm{i} b$ and from $s_{-}$ to $s_{-}-\mathrm{i} b$ on the non-principal Riemann sheet, and the horizontal line connecting the points $s_{+}-\mathrm{i} b$ and $s_{-}-\mathrm{i} b$, once again on the non-principal Riemann sheet. Here $b>0$. To calculate the integral along this contour we need to find the poles of the integrand inside this contour. These poles coincide with the zeros of Eq. (A.8). Since the area enclosed by the contour is on the non-principal Riemann sheet, we look for zeros of Eq. (A.8) on this sheet.

Although $w(s)$ is now not given by Eq. (A.7) but by Eq. (A.17), the roots of Eq. (A.8) are still close to either 0 or 1 or to one of the numbers $\pm s_{ \pm}$. Obviously, the root close to 0 cannot be inside the contour. Simple analysis shows that there are roots close to $\pm s_{-}$and $\pm s_{+}$, but their real parts are equal to $\pm \bar{s}_{-}$ and $\pm \bar{s}_{+}$, respectively, so these roots are also not inside the contour. Finally, the root close to 1 is given by

$s=\bar{s} \equiv 1-\frac{\mathrm{i} \pi \epsilon K}{8}$.

This root is inside the contour. Hence,

$\oint \frac{\mathrm{e}^{-\mathrm{i} s \omega t} \mathrm{~d} s}{2-2 s^{2}-\epsilon K \mathcal{L}[G(t)](s)}=$
$-2 \pi \operatorname{ires}_{s=\bar{s}} \frac{\mathrm{e}^{-\mathrm{i} s \omega t} \mathrm{~d} s}{2-2 s^{2}-\epsilon K \mathcal{L}[G(t)](s)}$.

We estimate the integrals along the parts of the closed contour that are on the non-principal Riemann sheet. Using the integration by parts we have

$$
\begin{aligned}
& \int_{s_{-}}^{s_{-}-\mathrm{i} b} \frac{\mathrm{e}^{-\mathrm{i} s \omega t} \mathrm{~d} s}{2-2 s^{2}-\epsilon K \mathcal{L}[G(t)](s)}= \\
& \int_{0}^{b} \frac{\exp \left[-\omega t\left(\sigma+\mathrm{i} s_{-}\right)\right] \mathrm{d} s}{2 \mathrm{i}-2 \mathrm{i}\left(s_{-}-\mathrm{i} \sigma\right)^{2}-\mathrm{i} \epsilon K \mathcal{L}[G(t)]\left(s_{-}-\mathrm{i} \sigma\right)} \rightarrow \frac{\mathrm{e}^{-\mathrm{i} \omega t s_{-}}}{\omega t} \int_{0}^{\infty} \mathrm{e}^{-\omega t \sigma} \\
& \times \frac{\mathrm{d}}{\mathrm{d} \sigma}\left(\frac{1}{2 \mathrm{i}-2 \mathrm{i}\left(s_{-}-\mathrm{i} \sigma\right)^{2}-\mathrm{i} \epsilon \kappa \mathcal{L}[G(t)]\left(s_{-}-\mathrm{i} \sigma\right)}\right) \mathrm{d} \sigma
\end{aligned}
$$


M. S. Ruderman and J. Terradas: Damping of coronal loop kink oscillations

where the limit is taken as $b \rightarrow \infty$. In the same way we prove that the integral along the vertical line connecting the points $s_{+}-$ $\mathrm{i} b$ and $s_{+}$is of the order of $1 / \omega t$. Finally, the integral along the horizontal line connecting the points $s_{-}-\mathrm{i} b$ and $s_{+}-\mathrm{i} b$ tends to zero as $b \rightarrow \infty$. As a result we have

$\int_{s_{+}}^{s_{-}} \frac{\mathrm{e}^{-\mathrm{i} s \omega t} \mathrm{~d} s}{2-2 s^{2}-\epsilon \kappa \mathcal{L}[G(t)](s)}=\widetilde{C}^{-\mathrm{i} \omega t} \exp \left(-\frac{\pi \epsilon \kappa \omega t}{8}\right)+O(1 / \omega t),(\mathrm{A} .22)$ where $\widetilde{C}$ is a complex constant. In a similar way we can obtain a similar expression for the integral along the interval $\left[-s_{-},-s_{+}\right]$.

It is worth explaining why we cannot prove in the same way that the integral along the interval $\left[s_{+}, s_{-}\right]$is of the order of $1 / \omega t$.
The reason is that there is a pole at $s=\bar{s}$, which is close to the interval $\left[s_{+}, s_{-}\right]$. After the integration by parts we obtain $1 / \omega t$ times an integral. The integrand in this integral is very big, in the vicinity of $s=1$, so the whole product is not of the order of $1 / \omega t$.

Summarising the analysis of this section, we arrive at the asymptotic expression

$\eta(t)=C \mathrm{e}^{-\mathrm{i} \omega t} \exp \left(-\frac{\pi \epsilon \kappa \omega t}{8}\right)+$ c.c. $+O(1 / \omega t)$,

where $C$ is a complex constant and c.c. denotes complex conjugate. 